Preprint typeset in JHEP style. - HYPER VERSION

AstroUSl-2000-1

\title{
The neutron star in the Relativistic Mean-Field Theory
}

\author{
by Ryszard Mańka *, llona Bednarek†, Grzegorz Przybyła \\ University of Silesia, Institute of Physics, Katowice 4000\%, ul. Uniwersytecka 4, \\ Poland.
}

\begin{abstract}
The nucleon effective mass, binding energy and neutron star configuration in the Relativistic Mean-Field Theory (RMF) is considered. The calculation are motivated by the construction of the equation of state for a neutron star in the RMF. The equation of state for the parameters set TM1 is calculated using the Feynman - Bogolubov variational method for temperature different from zero. The structure of the neutron star is presented. The maximal stable configuration are obtained for: $M_{\max }=1.91 M_{\odot}, \quad R=12.84 \mathrm{~km}$.
\end{abstract}

*email: manka@us.edu.pl

$\dagger$ email: bednarek@us.edu.p] 


\section{Introduction}

The physics of compact objects like neutron stars offers an intriguing interplay between nuclear processes and astrophysical observables. Neutron stars exhibit conditions far from those encountered on earth. The determination of an equation of state $(\mathrm{EoS})$ for dense matter is essential to calculations of neutron star properties.

This paper presents a basic model of neutron star [1] matter including interactions

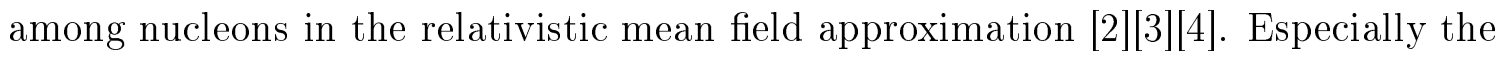
Walecka model (QHD) and its nonlinear extensions have been quite successful and widely used for the description of hadronic matter and finite nuclei. Increasing interest in neutron matter at finite temperature has been observed recently in relation to the problems of hot neutron stars and of protoneutron stars and their evolutions in particular. Theories concerning protoneutron stars are being discussed in works by Prakash et. al. [5]. Recently a detail calculation has been done with different models to study the properties of neutron stars [5]. Glendenning [6] has studied the properties of neutron star in the framework of nuclear relativistic field theory. In our calculations, we used the TM1 [0] parameter set, which has a capability to reproduce the known results of finite nuclei as well as of normal nuclear matter. The TM1 model possess a distinctively stiffer EOS.

\section{The Relativistic Mean Field Theory}

The fields of the model RMF for $\sigma, \omega$ and $\rho$-mesons are denoted as $\varphi, \omega_{\mu}, \rho_{\mu}$. The Lagrange density function for this model has the following form

$$
\begin{aligned}
\mathcal{L}= & \frac{1}{2} \partial_{\mu} \varphi \partial^{\mu} \varphi-\frac{1}{4} R_{\mu \nu}^{a} R^{a \mu \nu}-\frac{1}{4} F_{\mu \nu} F^{\mu \nu}+\frac{1}{2} M_{\omega}^{2} \omega_{\mu} \omega^{\mu}+\frac{1}{2} M_{\rho}^{2} \rho_{\mu}^{a} \rho^{a \mu} \\
- & U(\varphi)+\frac{1}{4} c_{3}\left(\omega_{\mu} \omega^{\mu}\right)^{2}+i \bar{\psi} \gamma^{\mu} D_{\mu} \psi-\bar{\psi}\left(M-g_{s} \varphi\right) \psi+ \\
& i \sum_{f=1}^{2} \overline{L_{f}} \gamma^{\mu} \partial_{\mu} L_{f}-\sum_{f=1}^{2} g_{f}\left(\bar{L}_{f} H e_{R f}+\text { h.c. }\right)
\end{aligned}
$$

where

$$
\begin{gathered}
R_{\mu \nu}^{a}=\partial_{\mu} \rho_{\nu}^{a}-\partial_{\nu} \rho_{\mu}^{a}+g \varepsilon_{a b c} \rho_{\mu}^{b} \rho_{\nu}^{c} \\
F_{\mu \nu}=\partial_{\mu} \omega_{\nu}-\partial_{\nu} \omega_{\mu} \\
D_{\mu}=\partial_{\mu}+\frac{1}{2} i g_{\rho} \rho_{\mu}^{a} \sigma^{a}+i g_{\omega} \omega_{\mu}
\end{gathered}
$$

The potential is given by

$$
U(\varphi)=\frac{1}{2} m_{s}^{2} \varphi^{2}-\frac{1}{3} g_{2} \varphi^{3}-\frac{1}{4} g_{3} \varphi^{4}=\frac{1}{2} m_{s}^{2} \varphi^{2}+\frac{1}{3 !} \kappa \varphi^{3}+\frac{1}{4 !} \lambda \varphi^{4}
$$




\begin{tabular}{|c|c|c|c|c|}
\hline Parameter & L2[3] & NBL[3] & LN1[12] & TM1[]] \\
\hline \hline$M$ & $938 \mathrm{MeV}$ & $938 \mathrm{MeV}$ & $938 \mathrm{MeV}$ & $938 \mathrm{MeV}$ \\
\hline$M_{w}$ & $786 \mathrm{MeV}$ & $786 \mathrm{MeV}$ & $795.359 \mathrm{MeV}$ & $783 \mathrm{MeV}$ \\
\hline$M_{\rho}$ & $770 \mathrm{MeV}$ & $770 \mathrm{MeV}$ & $763 \mathrm{MeV}$ & $770 \mathrm{MeV}$ \\
\hline$m_{s}$ & $500 \mathrm{MeV}$ & $510 \mathrm{MeV}$ & $492 \mathrm{MeV}$ & $511.198 \mathrm{MeV}$ \\
\hline$g_{2}=\kappa / 2$ & 0 & $2.03 \mathrm{fm}^{-1}$ & $12.172 \mathrm{fm}^{-1}$ & $7.2325 \mathrm{fm}^{-1}$ \\
\hline$g_{3}=\lambda / 6$ & 0 & 1.666 & -36.259 & 0.6183 \\
\hline$g_{s}$ & 10.0773 & 9.6959 & 10.138 & 10.0289 \\
\hline$g_{\omega}$ & 13.8655 & 12.5889 & 13.285 & 12.6139 \\
\hline$g_{\rho}$ & 8.48784 & 8.544 & 4.6322 & 4.6322 \\
\hline$c_{3}$ & 0 & 0 & 0 & 71.3075 \\
\hline
\end{tabular}

Table 1: Parameters Set for the Lagrangian (11).

The fermion fields are composed of protons, neutrons and electrons, muons and neutrinos

$$
\psi=\left(\begin{array}{c}
\psi_{p} \\
\psi_{n}
\end{array}\right), \quad L_{1}=\left[\begin{array}{c}
\nu_{e} \\
e^{-}
\end{array}\right]_{L}, L_{2}=\left[\begin{array}{l}
\nu_{\mu} \\
\mu^{-}
\end{array}\right]_{L}, e_{R f}=\left(e_{R}^{-}, \mu_{R}^{-}\right)
$$

$M$ is the nucleon mass and $m_{s}, M_{\omega}, M_{\rho}$ are masses assigned to the mesons fields, $g, g^{\prime}$ and $g_{s}$ are coupling constants. The Lagrangian function includes also the nonlinear term $\frac{1}{4} c_{3}\left(\omega_{\mu} \omega^{\mu}\right)^{2}$ which affects remarkably the form of the equation of state. the Higgs field $H$ takes the form of

$$
H=\frac{1}{\sqrt{2}}\left(\begin{array}{l}
0 \\
V
\end{array}\right)
$$

takes here the residual form. The parameters used in NBL model [3] are $m_{s}=500$ $\mathrm{MeV}$ with $\kappa=800 \mathrm{MeV}$ and $\lambda=10$. The Euler equation for $\Phi_{A}=\left\{\varphi, \omega_{\mu}, \rho_{\mu}, \psi\right\}$ fields are

$$
\begin{gathered}
\square \varphi=m_{s}^{2} \varphi+g_{2} \varphi^{2}+g_{3} \varphi^{3}-g_{s} \bar{\psi} \psi \\
-\partial_{\mu} F^{\mu \nu}=M_{\omega}^{2} \omega^{\nu}+c_{3}\left(\omega_{\mu} \omega^{\mu}\right) \omega^{\nu}-g_{\omega} J_{B}^{\nu}
\end{gathered}
$$

where

$$
J_{B}^{\nu}=\bar{\psi} \gamma^{\nu} \psi
$$

is the baryon current

$$
-D_{\mu} R^{\mu \nu, a}=M_{\rho}^{2} \rho^{\nu, a}-g_{\rho} J_{3}^{\nu}
$$

and

$$
J_{3}^{\nu}=\frac{1}{2} \bar{\psi} \gamma^{\nu} \sigma^{3} \psi
$$




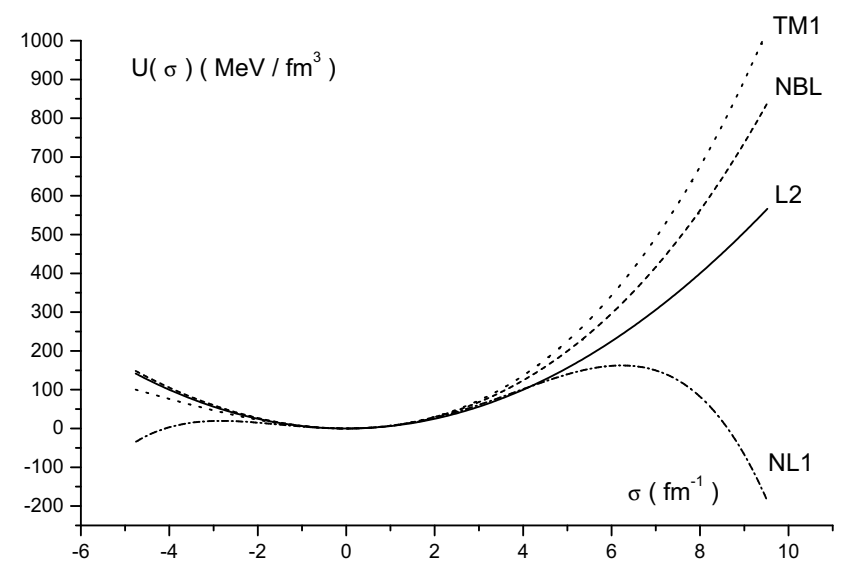

Figure 1: The potential $U(\varphi)$ in the relativistic mean field theory for L2 [3], NBL[3], LN1 [12], TM1|[0] phenomenological parameters.

is the isospin current. In the system we have conservation of baryon charge

$$
Q_{B}=\int d^{3} x J^{0}
$$

and the isospin charge

$$
Q_{3}=\int d^{3} x J_{3}^{0}
$$

The last is the Dirac equation

$$
i \gamma^{\mu} D_{\mu} \psi-\left(M-g_{s} \varphi\right) \psi=0 .
$$

The physical system is totally defined by the thermodynamic potential[ 8

$$
\Omega=-k \operatorname{Tln} \operatorname{Tr}\left(e^{-\beta\left(H-\mu Q_{B}-\mu_{3} Q_{3}\right)}\right)
$$

where $\mathrm{H}$ is the Hamiltonian of the physical system

$$
H=\sum_{A} \int d^{3} x\left\{\partial_{0} \Phi_{A} \pi_{\Phi}^{A}-\mathcal{L}\right\}
$$

and $\pi^{A}=\frac{\partial \mathcal{L}}{\partial\left(\partial_{0} \Phi_{A}\right)}$ is a momentum connected to the field $\Phi_{A}$. The fields $\Phi_{A}=\left\{\varphi, \omega_{\mu}\right.$, $\left.\rho_{\mu}, \psi\right\}$ denote all fields in the system. The average charges

$$
\frac{\partial \Omega}{\partial \mu}=-<Q_{B}>, \quad \frac{\partial \Omega}{\partial \mu_{3}}=-<Q_{3}>
$$

can be obtained from the thermodynamic potential, of course they should be conserved. In this paper we shall use the effective potential approach build using the Bogolubov inequality [9]

$$
\Omega \leq \Omega_{1}=\Omega_{0}\left(m_{B}, m_{F}\right)+<H-H_{0}>_{0}
$$


$\Omega_{0}$ is the thermodynamic potential of the trial system as effectively free quasiparticle system described by the Lagrange function

$$
\begin{aligned}
\mathcal{L}_{0}\left(m_{B}, m_{F}\right)= & \frac{1}{2} \partial_{\mu} \bar{\varphi} \partial^{\mu} \bar{\varphi}-\frac{1}{2} m_{B}^{2} \bar{\varphi}^{2}-\frac{1}{4} \bar{G}_{\mu \nu}^{a} \bar{G}^{a \mu \nu}-\frac{1}{4} \bar{F}_{\omega \cdot \mu \nu} \bar{F}_{\omega}^{\mu \nu} \\
& +\frac{1}{2} M_{\omega}^{2} \bar{\omega}_{\mu} \bar{\omega}^{\mu}+\frac{1}{2} M_{\rho}^{2} \bar{\rho}_{\mu}^{a} \bar{\rho}^{a \mu}+\bar{\psi}\left(i \gamma^{\mu} \bar{D}_{\mu}-m_{F}\right) \psi
\end{aligned}
$$

Similar to the general case

$$
\bar{G}_{\mu \nu}^{a}=\partial_{\mu} \bar{\rho}_{\nu}^{a}-\partial_{\nu} \bar{\rho}_{\mu}^{a}
$$

and

$$
\bar{F}_{\omega, \mu \nu}=\partial_{\mu} \bar{\omega}_{\nu}-\partial_{\nu} \bar{\omega}_{\mu}
$$

We decompose the $\Phi_{A}$ field into two components, the effectively free quasiparticle field $\tilde{\Phi}_{A}$ and the classical boson condensate $\xi_{A}$

$$
\Phi_{A}=\tilde{\Phi}_{A}+\xi_{A}
$$

In the case of the RMF model we have

$$
\begin{gathered}
\varphi=\bar{\varphi}+\sigma \\
\omega_{\mu}=\bar{\omega}_{\mu}+w_{\mu}, w_{\mu}=\delta_{\mu, 0} w \\
\rho_{\mu}^{a}=\bar{\rho}^{a}+r_{\mu}^{a}, r_{\mu}^{a}=\delta^{a, 3} \delta_{\mu, 0} r
\end{gathered}
$$

The $\xi_{A}=\{\sigma, w, r\}$ field will be treated as the variational parameters in the effective potential. Also the boson and fermion mass $m_{B}, m_{F}$ will be treated as as the variational parameters. The covariant derivative for the trial system is

$$
\bar{D}_{\mu}=\partial_{\mu}+\frac{1}{2} i g_{\rho} r_{\mu}^{a} \sigma^{a}+i g_{\omega} w_{\mu}
$$

This introduce the homogenous fermion interaction with boson condensate $w_{\mu}, r_{\mu}^{a}$. The fermion quasiparticle will obey the Dirac equation

$$
\left(i \gamma^{\mu} \bar{D}_{\mu}-m_{F}\right) \psi=0
$$

The constant condensate $w, r$ simply shift the chemical potential from $\mu_{i}=\mu_{i}^{0}$ (when $w=r=0)$ to

$$
\begin{aligned}
& \mu_{n}=\mu_{n}^{0}+\frac{1}{2} g_{\rho} r-g_{\omega} w \\
& \mu_{p}=\mu_{p}^{0}-\frac{1}{2} g_{\rho} r-g_{\omega} w
\end{aligned}
$$

where $\mu_{n}=\mu+\frac{1}{2} \mu_{3}$ and $\mu_{p}=\mu-\frac{1}{2} \mu_{3}$.

Neutrons, protons and electrons are in $\beta$-equilibrium which can be described as a relation among their chemical potentials

$$
\mu_{p}+\mu_{e}=\mu_{n}
$$


where $\mu_{p}, \mu_{n}$ and $\mu_{e}$ stand for proton, neutron and electron chemical potentials respectively. If the electron Fermi energy is high enough (greater then the muon mass) in the neutron star matter muons start to appear as a result of the following reaction

$$
e^{-} \rightarrow \mu^{-}+\nu_{e}+\overline{\nu_{\mu}}
$$

The chemical equilibrium between muons and electrons can be described by the condition

$$
\mu_{\mu}=\mu_{e}
$$

When neutrinos are trapped inside the protoneutron star also the neutrino chemical potential should be included

$$
\mu_{p}+\mu_{e}=\mu_{n}+\mu_{\nu_{e}}
$$

The density of the thermodynamic potential $f_{1}=\Omega_{1} / V$ is equal to

$$
\begin{gathered}
f_{1}\left(m_{B}, m_{F}, \sigma, w, r\right)= \\
f_{0}\left(m_{B}, m_{F}\right)+\frac{1}{2}<\bar{\varphi}^{2}>_{0}\left(m_{s}^{2}-m_{B}^{2}\right)+\frac{1}{8} \lambda<\bar{\varphi}^{2}>_{0}^{2}+ \\
\frac{1}{2} \kappa<\bar{\varphi}^{2}>_{0} \sigma+\frac{1}{2}\left(m_{s}^{2}+\frac{1}{2} \lambda<\bar{\varphi}^{2}>_{0}\right) \sigma^{2}+ \\
\frac{1}{3 !} \kappa \sigma^{3}+\frac{1}{4 !} \lambda \sigma^{4}+<\bar{\psi} \psi>_{0}\left(g \sigma-m_{F}\right)+. . \\
f_{0}=f_{B}+f_{F}
\end{gathered}
$$

where $f_{B}$ is the boson free energy and $f_{F}$ the fermion one. For boson field the free energy is

$$
f_{B}=\frac{k_{B} T}{(2 \pi)^{3}} \int d^{3} p \ln \left(1-e^{-\beta \omega(p)}\right)
$$

with $\omega(p)=\sqrt{\mathbf{p}^{2}+m_{B}^{2}}$. For fermions we have 4 degree of freedom, 2 for spin $1 / 2$ and 2 for particle - antiparticle distinguishing. For one fermion field the free energy is equal to

$$
f_{F}=-\sum_{i=\{n, p\}} \frac{2 k_{B} T}{(2 \pi)^{3}} \int d^{3} p\left\{\ln \left(1+e^{-\beta\left(\epsilon(p)-\mu_{i}\right)}\right)+\ln \left(1+e^{-\beta\left(\epsilon(p)+\mu_{i}\right)}\right)\right\}
$$

now with $\epsilon(p)=\sqrt{\mathbf{p}^{2}+m_{F}^{2}}$. Variation

$$
\frac{\partial f_{1}}{\partial m_{B}^{2}}=0, \frac{\partial f_{1}}{\partial m_{F}}=0
$$

with respect to the trial system $L_{0}$ gives

$$
m_{B}^{2}=m_{s}^{2}+2 g_{2} \sigma+3 g_{3}\left(\sigma^{2}+<\bar{\varphi}^{2}>_{0}\right),
$$




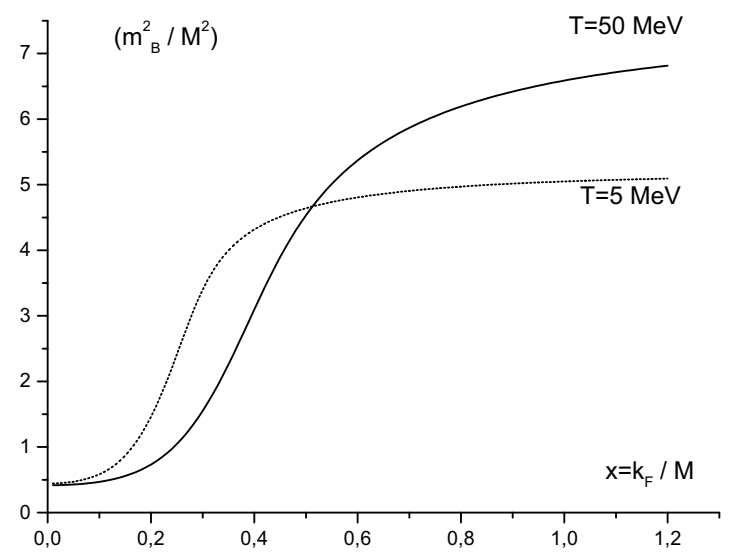

Figure 2: Meson mass $m_{B}^{2} / M^{2}$ dependence on the Fermi momentum $x=k_{F} / M$ for temperature $T=50 \mathrm{MeV}$.

$$
m_{F}=M \delta=M-g_{s} \sigma .
$$

In the local equilibrium inside the star the free energy reaches the minimum at $\sigma$.

The same result may be achieved calculating the averages of the equation of motions (9) for the effective system $\mathcal{L}_{0}$. In the mean field approximation the meson field operators are replaced by their expectation values. We also consider the isotropic system at rest. For the scalar field the equation (9) have is follows

$$
\left(m_{s}^{2}+\frac{1}{2} \lambda<\bar{\varphi}^{2}>_{0}\right) \sigma+\frac{1}{2} \kappa \sigma^{2}+\frac{1}{6} \lambda \sigma^{3}=g_{s}<\bar{\psi} \psi>_{0}-\frac{1}{2} \kappa<\bar{\varphi}^{2}>_{0}
$$

If we shall notice that

$$
\frac{\partial f_{B}}{\partial m_{B}^{2}}=\frac{1}{2}<\bar{\varphi}^{2}>_{0}
$$

then it easy to obtain

$$
<\bar{\varphi}^{2}>_{0}=\frac{1}{2 \pi^{2}} \int \frac{d p p^{2}}{\sqrt{p^{2}+m_{B}^{2}}} \frac{1}{(\exp (\beta \omega(p))-1)}
$$

This result means that equation (36) is highly nonlinear with respect to the meson mass $m_{B}$ (Fig. 2). As $<\bar{\psi} \psi>_{0}$ (calculated with respect to $\mathcal{L}_{0}$ system) depends on the effective nucleon mass $m_{F}$ (or $\sigma$ ) the equation (38) is highly nonlinear also with respect to $\sigma$. In most of papers [10] temperature dependence of the boson fields is neglected. Contrary to the fermion case the effective mass of boson $m_{B}$ growing with Fermi momentum. In the result the bosons temperature contributions may be neglected.

Calculation similar to the boson case, base on the relation

$$
\frac{\partial f_{F}}{\partial m_{F}}=<\bar{\psi} \psi>_{0}
$$




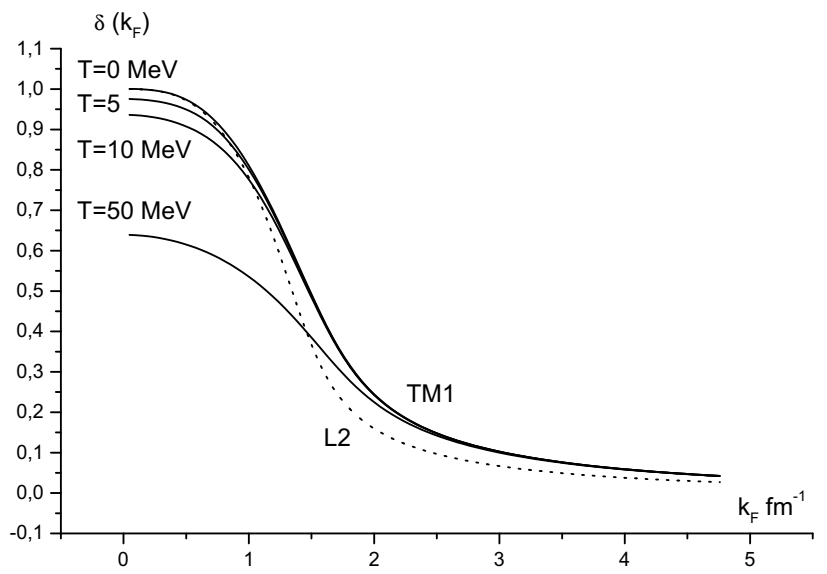

Figure 3: The effective neutron mass $m_{F}=\delta\left(x_{F}\right) M$ as function of the neutron Fermi momentum $x=k_{F}\left(f m^{-1}\right)$.

gives

$$
\begin{aligned}
<\bar{\psi} \psi>_{0}=\sum_{i=\{n, p\}} \frac{m_{F}}{\pi^{2}} \int_{0}^{\infty} \frac{p^{2} d p}{\sqrt{p^{2}+m_{F}^{2}}}\left\{\frac{1}{\exp \left(\beta\left(\epsilon_{p}-\mu_{i}\right)\right)+1}+\right. \\
\\
\left.\frac{1}{\exp \left(\beta\left(\epsilon_{p}+\mu_{i}\right)\right)+1}\right\} .
\end{aligned}
$$

The quantum average $<\bar{\psi} \psi>_{0}$ depends on the neutron and proton chemical potentials $\mu_{p}, \mu_{n}$. In the result the effective nucleon effective mass $m_{F}$ also will be dependent he neutron and proton chemical potentials. In the result the solution $\sigma$ of the equation (9) also will be dependent on $\xi$. The same situation will consider other fields. This model is the simple example of the relativistic mean filed theory [3].

The effective mass $m_{F}$ (or $\delta=m_{F} / M$ ) dependence on the dimensionless Fermi momentum $x_{F}$ is presented on the Fig. 3. The binding energy

$$
E_{0}=\epsilon\left(x_{F}, T\right) / Q_{B}-M
$$

for nucleon symmetric phase is presented on Fig. 2 (see Table 2). We see that binding energy strongly depends on temperature and above $T>15 \mathrm{MeV}$ is positive. The temperature dependence of the binding energy in presented on the Fig 4 . To calculate the properties of the neutron star we need the energy-momentum tensor. In case of the fermions field it is more convenient to use the reper filed $e_{\mu}^{a}$ defined as follows $g_{\mu \nu}=e_{\mu}^{a} e_{\nu}^{b} \eta_{a b}$ where $\eta_{a b}$ is the flat Minkowski space-time matrix. The general definition

$$
T_{\mu \nu}=2 \frac{\partial L_{B}}{\partial g^{\mu \nu}}+e_{\mu}^{a} \frac{\partial L_{F}}{\partial e^{a \nu}}-g_{\mu \nu} L
$$




\begin{tabular}{|c|c|c|c|c|}
\hline Parameter & L2[司] & NBL[3] & LN1[12] & TM1[[] \\
\hline \hline$E_{0} \mathrm{MeV}$ & -15.74 & -16.59 & -16.42 & -16.26 \\
\hline$k_{F, 0} \mathrm{fm}^{-1}$ & 1.30 & 1.31 & 1.309 & 1.29 \\
\hline$\rho_{0} \mathrm{fm}^{-3}$ & 0.149 & 0.1524 & 0.1517 & 0.1452 \\
\hline$\delta_{0}=m_{F} / \mathrm{M}$ & 0.54 & 0.60 & 0.573 & 0.634 \\
\hline $\mathrm{K} \mathrm{MeV}$ & 545.93 & 445.73 & 215.19 & 281.53 \\
\hline
\end{tabular}

Table 2: The nucleon symmetric phase properties at the saturation point.

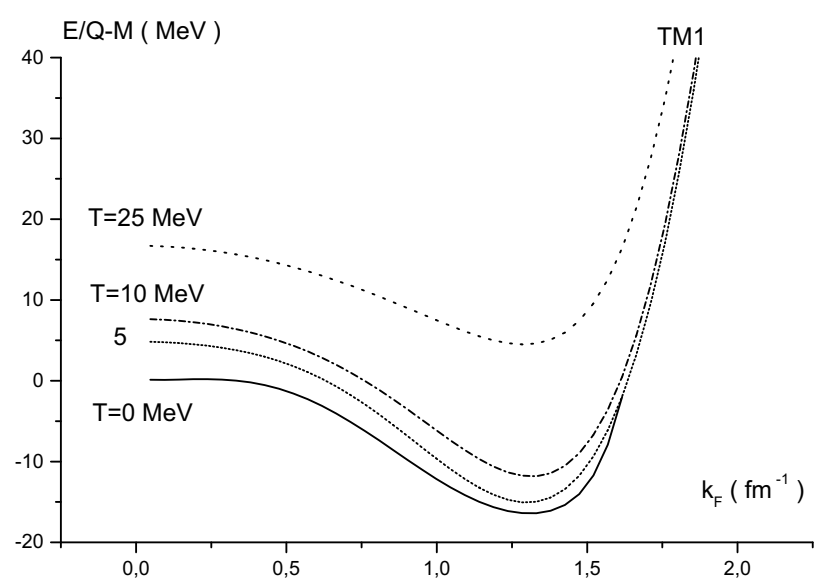

Figure 4: The temperature dependence of the binding energy for nucleon symmetric TM1 phase.

allows us to calculate the density of energy and pressure. The total Lagrange function $L=L_{B}+L_{F}$ is divided into boson and fermion part. To calculate the density of energy and pressure we shall average the energy-momentum tensor $T_{\mu \nu}$ with respect to the quasi equilibrium configuration defined by the trial system $L_{0}$. We define the density of energy and pressure by the energy - momentum tensor

$$
<T_{\mu \nu}>=(P+\epsilon) u_{\mu} u_{\nu}-P g_{\mu \nu}
$$

where $u_{\mu}$ is a unite vector $\left(u_{\mu} u^{\mu}=1\right)$. So, the calculations give

$$
\begin{gathered}
\epsilon\left(x_{F}, T\right)=\rho c^{2}=-\frac{1}{2} M_{\omega}^{2} w^{2}-\frac{1}{2} M_{\rho}^{2} r^{2}+g_{\omega} Q_{B} w+\frac{1}{2} g_{\rho} Q_{3} r-\frac{1}{4} c_{3} w^{4}+U(\sigma)+\epsilon_{F} \\
P\left(x_{F}, T\right)=\frac{1}{2} M_{\omega}^{2} w^{2}+\frac{1}{2} M_{\rho}^{2} r^{2}+\frac{1}{4} c_{3} w^{4}-U(\sigma)+P_{F}
\end{gathered}
$$

where

$$
\begin{gathered}
\epsilon_{F}=\epsilon_{0} \chi\left(x_{F}, T\right) \\
P_{F}=P_{0} \phi\left(x_{F}, T\right)
\end{gathered}
$$




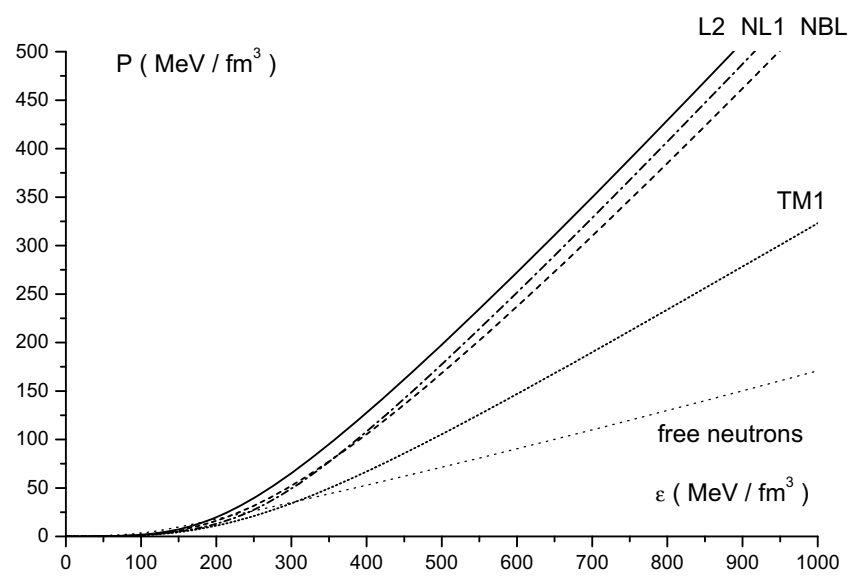

Figure 5: The equation of state for different parameters sets (L2, NBL,TM1).

The fact that neutron mass depends on fermion concentration ( or neutron chemical potential $\mu$ ) now must be included into $\chi\left(x_{F}, T\right)$ and $\phi\left(x_{F}, T\right)$,

$$
\begin{array}{r}
\chi\left(x_{F}, T\right)=\frac{1}{\pi^{2}} \int_{0}^{\infty} d z z^{2} \sqrt{z^{2}+\delta^{2}\left(x_{F}\right)}\left\{\frac{1}{\exp \left(\left(\sqrt{\delta^{2}\left(x_{F}\right)+z^{2}}-\mu^{\prime}\right) / \tau\right)+1}\right. \\
+\frac{1}{\exp \left(\left(\sqrt{\delta^{2}\left(x_{F}\right)+z^{2}}+\mu^{\prime}\right) / \tau\right)+1}, \\
\phi\left(x_{F}, T\right)=\frac{1}{3 \pi^{2}} \int_{0}^{\infty} \frac{z^{4} d z}{\sqrt{z^{2}+\delta^{2}\left(x_{F}\right)}}\left\{\frac{1}{\exp \left(\left(\sqrt{\delta^{2}\left(x_{F}\right)+z^{2}}-\mu^{\prime}\right) / \tau\right)+1}\right. \\
\left.+\frac{1}{\exp \left(\left(\sqrt{\delta^{2}\left(x_{F}\right)+z^{2}}+\mu^{\prime}\right) / \tau\right)+1}\right\}
\end{array}
$$

where $\tau=\left(k_{B} T\right) / M$,

$$
\mu^{\prime}=\mu / M=\sqrt{\delta^{2}\left(x_{F}\right)+x_{F}^{2}}
$$

and

$$
x_{F}=k_{F} / M
$$

Similar to paper 112 we have introduced (49,50) the dimensionless "Fermi" momentum even at finite temperature which exactly corresponds to the Fermi momentum at zero temperature. Both $\epsilon_{F}$ and $P_{F}$ depend on the neutron chemical potential $\mu$ or Fermi momentum $x_{F}$. This parametric dependence on $\mu$ (or $x_{F}$ ) defines the equation of state. The various equations of state for different parameters sets is presented on fig.5. The equation of state for the parameters set TM1 for temperature $\mathrm{T}=10 \mathrm{MeV}$ 


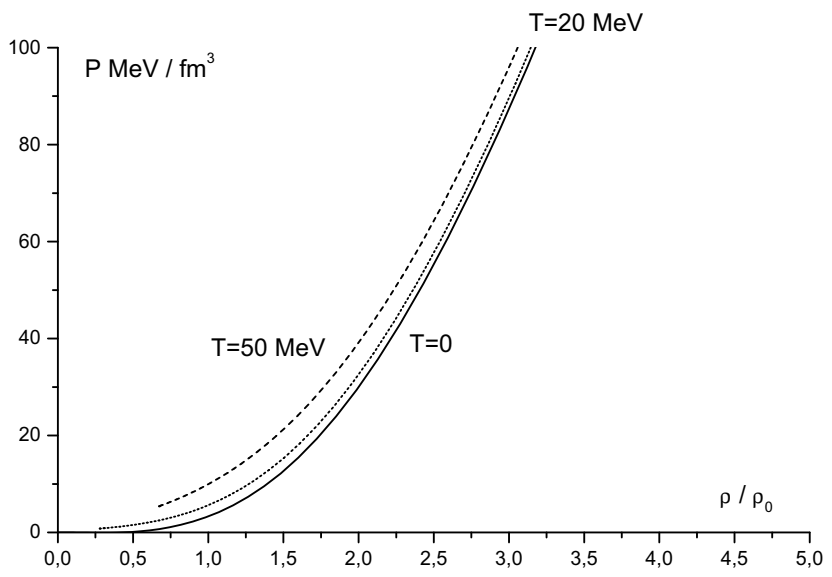

Figure 6: The equation of state for the parameters set TM1 for $\mathrm{T}=0$ (solid line) and $\mathrm{T}=20,50 \mathrm{MeV}$ temperature.

is presented on fig.6. It is interesting to notice that even for $Q_{B}=0$ due to the presence of the thermal exited particle antiparticle pairs there is finite energy and pressure density. It is interesting to notice that the meson field $\varphi$ as the scalar field contributes to the pressure with negative sign while the vector meson fields $(\omega, \rho)$ with the positive one.

\section{The neutron star}

In this paper we present numerical results describing the structure of neutron star based on the relativistic mean field theory. It is possible to describe a static spherical star solving the OTV equation.

$$
\begin{gathered}
\frac{d P(r)}{d r}=-\frac{G}{r^{2}}\left(\rho(r)+\frac{P(r)}{c^{2}}\right) \frac{\left(m(r)+\frac{4 \pi}{c^{2}} P(r) r^{3}\right)}{\left(1-\frac{2 G m(r)}{c^{2} r}\right)} \\
\frac{d m(r)}{d r}=4 \pi r^{2} \rho(r)
\end{gathered}
$$

Having solved the OTV equation the pressure $p(r)$, mass $m(r)$ and density $\rho(r)$ were obtained. To obtain the total radius $R$ of the star the fulfillment of the condition $p(R)=0$ is necessary. This allows to determine the total gravitational mass of the star $M(R)$.

Introducing the dimensionless variable $\xi$, which is connected with the star radius $r$ by the relation $r=a \xi$ enables to define the functions $p(r), \rho(r)$ and $m(r)$

$$
\rho(r)=\rho_{0} \chi(x(\xi))
$$




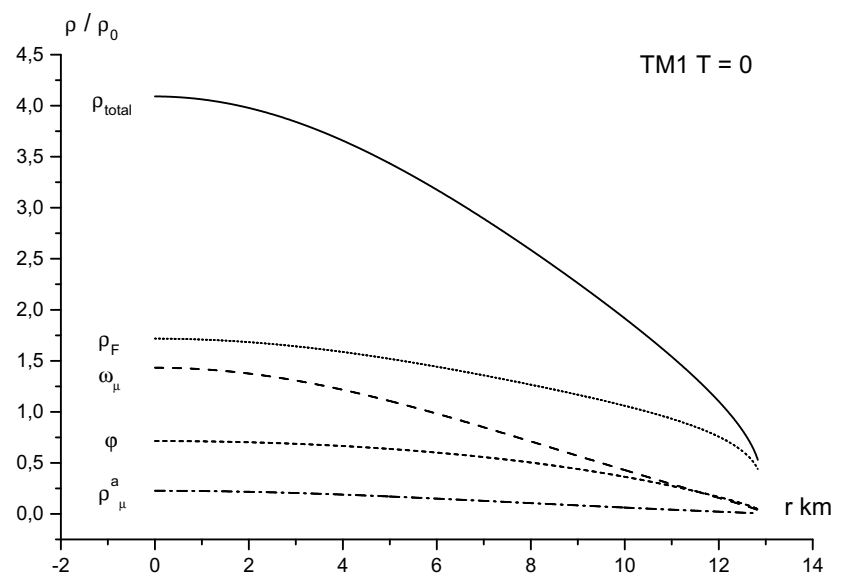

Figure 7: The neutron star density profile for fermions $\rho_{F}$ scalar bosons $\varphi$ and gauge bosons $\left(\omega_{\mu}, \rho_{\mu}^{a}\right)$.

$$
\begin{gathered}
P(r)=P_{0} \varphi(x(\xi)) \\
m(r)=M_{\odot} v(\xi)
\end{gathered}
$$

by $\xi$. If we define dimensionless functions.

$$
\lambda=\frac{G M_{\odot} \rho_{c}}{P_{0} a}, \mu=3 \frac{M_{c}}{M_{\odot}}, M_{c}=\frac{4}{3} \pi \rho_{0} a^{3}
$$

are also need to achieve the OTV equation of the following form

$$
\begin{gathered}
\frac{d \varphi}{d \xi}=-\lambda(\chi(\xi)+\varphi(\xi)) \frac{v(\xi)+\mu \varphi(\xi) \xi^{3}}{\xi^{2}\left(1-\frac{r_{g}}{a} \frac{v(\xi)}{\xi}\right)} \\
\frac{d v}{d \xi}=\mu \chi(\xi) \xi^{2}
\end{gathered}
$$

with $r_{g}$ being the gravitational radius. The equations $(57,58)$ are easy integrated numerically, For example, for the neutron star with the central density $\rho_{c}=910^{14} \mathrm{~g} / \mathrm{cm}^{3}$ the star profile in the mean field approach is presented on the Fig.7. It's interesting that fermions contribution to density is lower then a half of total density. The biggest contributions come from the nucleons, the gauge boson field $\omega_{\mu}$ and scalar boson field $\varphi$. On the surface of star we have non zero value of density $\left(\rho \sim 0.25<\rho_{0}\right)$ and nucleon mass lower than vacuum nucleon mass $\left(m_{F} \sim 762 \mathrm{MeV}<M\right)$. Inside star with $\rho_{c}=9 \times 10^{14} \mathrm{~g} / \mathrm{cm}^{3}$ (maximal stable configuration for the TM1 parameters set) value of nucleon mass varies from $\approx 300 \mathrm{MeV}$ in centrum of star to $\approx 762 \mathrm{MeV}$ on 


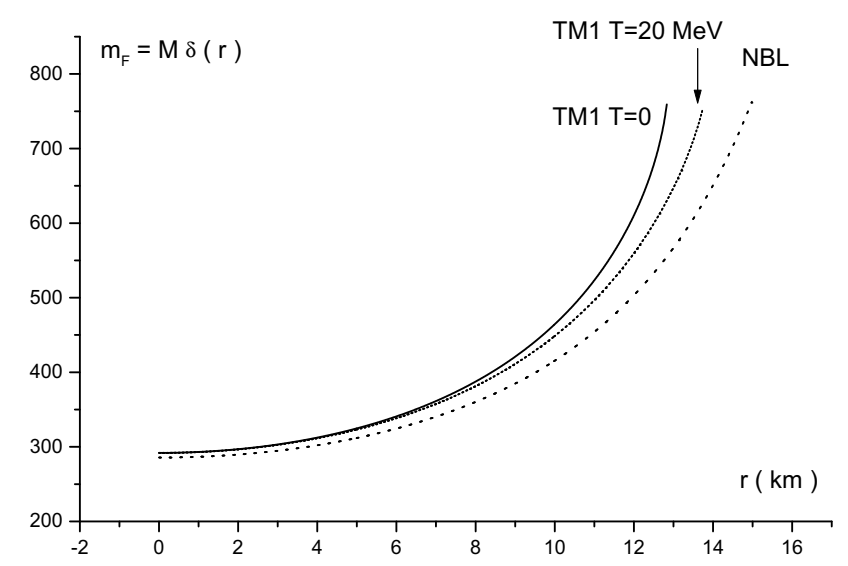

Figure 8: The effective neutron mass profile $\left(m_{N}=M \delta\right)$ inside the star.

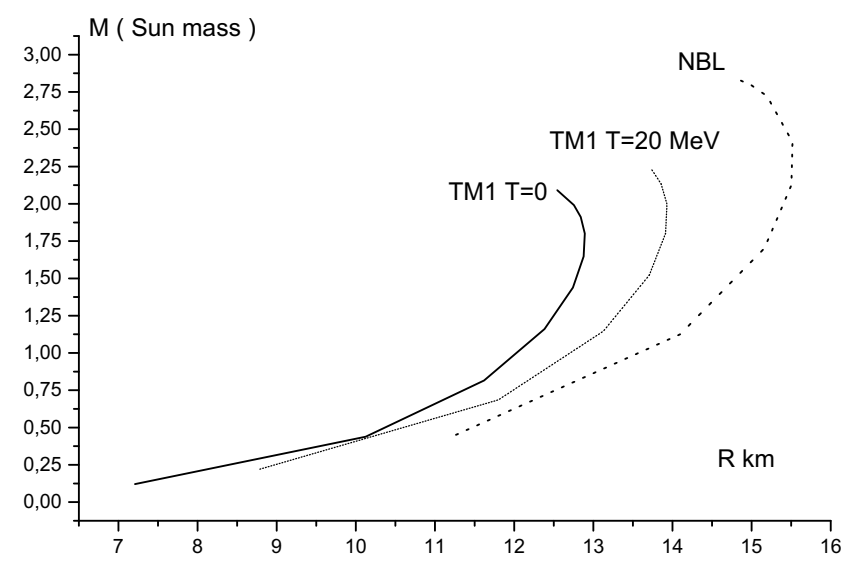

Figure 9: The R-M diagram for the neutron star.

the surface (Fig. 8). Fig. 10. shows the stellar masses as a function of the central density. The parameters of the maximum mass configuration are:

$$
M_{\max }=1.91 M_{\odot}, \quad R=12.84 \mathrm{~km} .
$$

This fact is easy to notice on the mass-radius diagram (Fig. 9). When temperature is different from zero, a star is in generar bigger and more massive (see Figs. 9 and 10).

\section{Conclusion}

The structure of static neutron stars can be determined by solving the Tolman- 


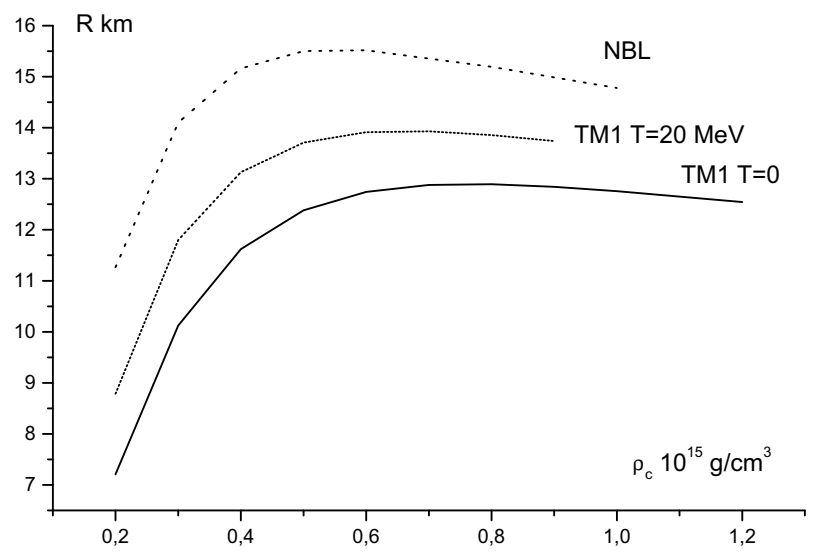

Figure 10: The neutron star radius dependence from the central density $\rho_{c}$.

Oppenheimer-Volkoff equations. The equation of state will strong influence on the neutron star properties. The aim of this work has been to present the equation of state in the RMF for the nuclear matter with temperature different from zero. The hot neutron star in the simple L2 parameters set was examined in our previous work [13]. The main objective of our work was to study the influence of the temperature on the main parameters of a neutron star. Neutron matter at finite temperature is of increasing interest in relation to the problems of hot neutron stars. In order to achieve the proper form of the equation of state the relativistic mean field approach was involved. 


\section{Appendix: Nuclear matter properties}

The nuclear symmetric matter may be described by the phenomenological equation of state [14]

$$
\varepsilon(u)=\rho_{0} u\left(M+\frac{3}{5} \frac{\hbar^{2} k_{F}^{2}}{2 M}+\frac{1}{2} A u^{2}+\frac{B}{(\sigma+1)} u^{\sigma}\right)
$$

where $u=\rho / \rho_{0}$ is a dimensionless density. For symmetric nuclear matter dimension-

less $\rho_{0}=2.510^{14} \mathrm{~g} \mathrm{~cm}^{-3}=0.15$ nucleons $/ \mathrm{fm}^{3}=140 \mathrm{MeV} \mathrm{fm}^{-3}$. The parameter of $\varepsilon(60)$ are

$$
\begin{gathered}
\sigma=\frac{K_{0}+2 E_{F, 0}}{3 E_{F, 0}-9 E_{0}}, \\
B=\left(\frac{\sigma+1}{\sigma-1}\right)\left[\frac{1}{3} E_{F, 0}-E_{0}\right], \\
A=E_{0}-\frac{5}{3} E_{F, 0}-B .
\end{gathered}
$$

$E_{F, 0}$ is the nonrelativistic nucleon Fermi energy at the saturation point $k_{F, 0}$ (see Table 2). For the TM1 parameter set (see Table 1) we have

$$
\sigma=1.5485 ; A=-1.58 .52 \mathrm{MeV} ; B=107.689 \mathrm{MeV} ;
$$

In this approach the binding energy (see Fig.11 ) is

$$
E_{0}(u)=E_{F, 0} u^{\frac{2}{3}}+\frac{1}{2} A u+\frac{B}{(\sigma+1)} u^{\sigma} .
$$

The pressure is

$$
P=\left.u^{2} \frac{d\left(\varepsilon / Q_{B}\right)}{d \rho}\right|_{\rho_{0}}=\frac{2}{3} E_{F, 0} u^{\frac{5}{3}}+\frac{1}{2} A u^{2}+\frac{B \sigma}{(\sigma+1)} u^{\sigma+1} .
$$

The minimum of the binding energy determine the equilibrium Fermi momentum $k_{F, 0}$, density $\rho_{0}$ and incompressibility factor

$$
K=\left.9 \rho_{0} \frac{d^{2}\left(\varepsilon / Q_{B}\right)}{d \rho^{2}}\right|_{\rho_{0}} .
$$

The empirical value of $K$ is $210 \pm 30 \mathrm{MeV}$ [11]. The incompressibility factor is equal to

$$
K(u)=\left.9 \frac{d(P / \rho)}{d u}\right|_{\rho_{0}}=10 E_{F, 0} u^{\frac{2}{3}}+9\left(A u+B \sigma u^{\sigma}\right) .
$$

The chemical potential defined for particle species $i$ is given by

$$
\mu_{i}(u)=\frac{d(\varepsilon)}{d \rho_{i} .} .
$$




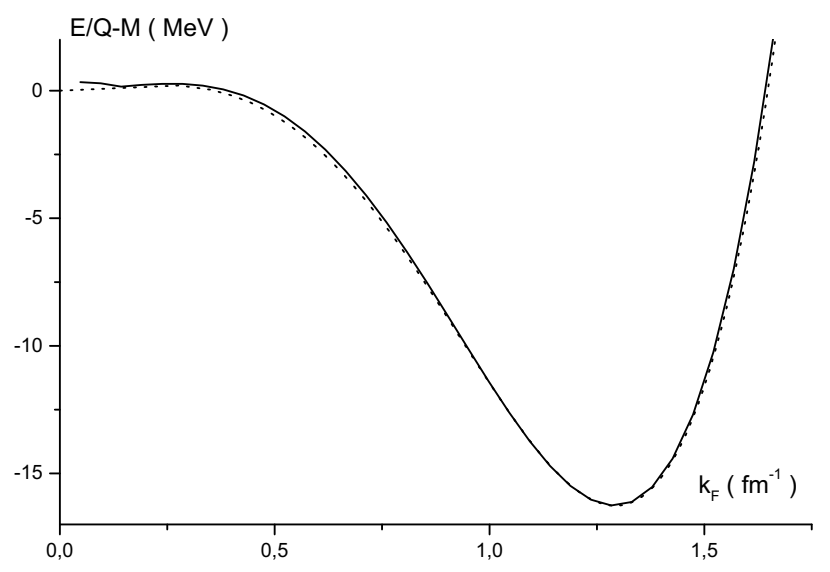

Figure 11: The binding energy for nucleon symmetric TM1 phase according to the RMF approach (the solid line) and (64) (the dot line), respectively.

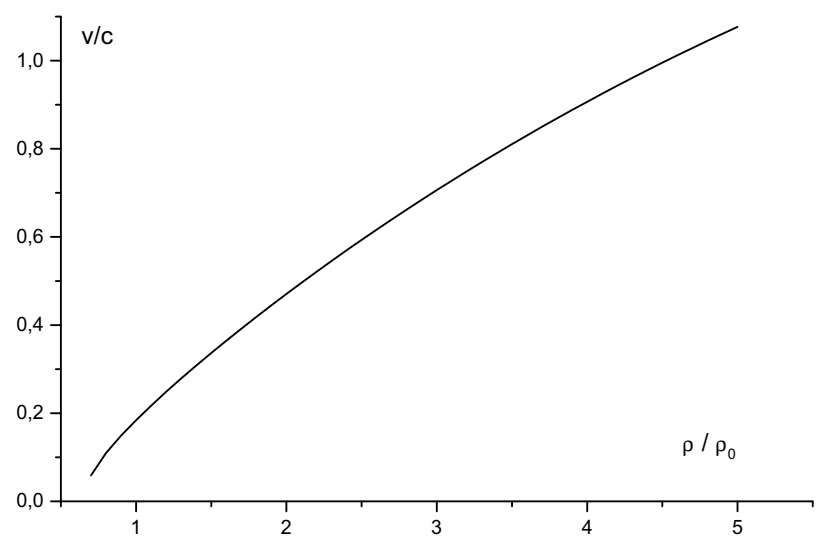

Figure 12: The adiabatic sound speed as the function of the relative density $u=\rho / \rho_{0}$ for the TM1 parameters set.

For example, for nucleon symmetric saturation point $\mu=912.73 \mathrm{MeV}$ for nucleons. The adiabatic sound speed

$$
\left(\frac{v}{c}\right)^{2}=\frac{d P}{d \varepsilon}=\frac{K}{9 \mu}
$$

is presented on the Fig.12. At the saturation point we have the sound speed $v=$ 0.184c. For TM1 parameters, for $\rho$ above $5 \rho_{0}$ the theory looses its sense. For other parameters sets when $K$ is grater the range of the theory validity is smaller. Acknowledgment

The authors are thankful to F. Weber for helpful discussions. 


\section{References}

[1] H.Heiselberg, Neutron star: recent developments, nucl-th/991202; H.Heiselberg, M.Hjorth-Jensen, Phases of Dense Matter in Neutron Stars, 1999.

[2] P.-G.Reinhard, M.Rufa, J.Maruhn,W.Greiner,J.Friedrich, Z. Phys. A - Atomic Nuclei 323,13-25, (1986).

[3] Serot B.D., Walecka J.D. Recent Progress in Quantum Hadrodynamics, Int. J. Mod. Phys. E6, 515-631, (1997)

[4] Weber F. Pulsars as Astrophysical Laboratories for Nuclear and Particle Physics, IOP Publishing, Philadelphia, (1999)

[5] Prakash M.et al. Phys. Reports, 280, 1-77, (1997)

[6] N.K. Glendenning, Z. Phys., A327, (1987) 295; Also see in Compact Stars by N. K Glendenning,( Sringer-Verlag, New York, 1997).

[7] Y. Sugahara and H. Toki, Prog. Theo. Phys. 92, (1994), 803

[8] A.L.Fetter, J.D.Walecka, Quantum Theory of Many-Particle Systems, McGraw-Hill, New York, 1971.

[9] R.Feynman, Statistical Mechanics, W.A.Benjamin, Inc. Reading 1972.

R.Mańka, J.Kuczyński, G.V.Vittiello, Nucl. Phys. B276, (1986),533; Annals of Physics, 199,R.Mańka, G.V.Vittiello, 199, (1990),61.

[10] H.Müller, B.D.Serot, Phase Transitions in the Warm, Asymmetric Nuclear Matter, nucl-th/9505013.

[11] J.Blaizot, D.Gogny and B.Grammaticos, Nucl. Phys. A265, (1975), 315.

[12] K.Sumiyoshi, H.Toki, ApJ, 422, 700,1994

[13] I.Bednarek, R.Mańka, Physica Scripta, im press, 2000, nucl-th/9912039.

[14] M.Prakash, The Equation of State and Neutron Star, lectures delivered at the Winter School held in Puri, India, 1994. 